\title{
Calculation of Wave Dispersion Curves in Multilayered Composite-Metal Plates
}

\author{
Ameneh Maghsoodi, ${ }^{1}$ Abdolreza Ohadi, ${ }^{1}$ and Mojtaba Sadighi ${ }^{2}$ \\ ${ }^{1}$ Acoustics Research Laboratory, Department of Mechanical Engineering, Amirkabir University of Technology, \\ 424 Hafez Avenue, Tehran 15916-34311, Iran \\ ${ }^{2}$ Department of Mechanical Engineering, Amirkabir University of Technology, 424 Hafez Avenue, Tehran 15916-34311, Iran
}

Correspondence should be addressed to Abdolreza Ohadi; a_r_ohadi@aut.ac.ir

Received 19 October 2012; Accepted 19 November 2012; Published 12 June 2014

Academic Editor: Hamid Mehdigholi

Copyright (C) 2014 Ameneh Maghsoodi et al. This is an open access article distributed under the Creative Commons Attribution License, which permits unrestricted use, distribution, and reproduction in any medium, provided the original work is properly cited.

\begin{abstract}
The major purpose of this paper is the development of wave dispersion curves calculation in multilayered composite-metal plates. At first, equations of motion and characteristic equations for the free waves on a single-layered orthotropic plate are presented. Since direction of wave propagation in composite materials is effective on equations of motion and dispersion curves, two different cases are considered: propagation of wave along an axis of material symmetry and along off-axes of material symmetry. Then, presented equations are extended for a multilayered orthotropic composite-metal plate using the transfer matrix method in which a global transfer matrix may be extracted which relates stresses and displacements on the top layer to those on the bottom one. By satisfying appropriate boundary conditions on the outer boundaries, wave characteristic equations and then dispersion curves are obtained. Moreover, presented equations may be applied to other materials such as monoclinic, transversely isotropic, cubic, and isotropic materials. To verify the solution procedure, a number of numerical illustrations for a single-layered orthotropic and double-layered orthotropic-metal are presented.
\end{abstract}

\section{Introduction}

Nondestructive inspection based on the propagation of elastic waves that relies generally on calculation of dispersion curves plays an important role in damage identification in multilayered structures. From this point of view, calculation of dispersion curves is one of the essential stages for inspection of structures.

Most of the researches have been done on the singlelayered isotropic or quasi-isotropic materials $[1,2]$. However, Nayfeh [3] has presented a transfer matrix technique to obtain the dispersion curves of elastic waves propagating in multilayered anisotropic media, that is, composite laminates. Demcenko and Mazeika [4] have developed global matrix method to calculate dispersion curves of multilayered isotropic plate. Lowe [5] has presented a review of development of the matrix method and global matrix method to obtain dispersion curves in isotropic materials. Also, they have discussed the problems with large value of frequency thickness, material damping, and leaky wave. Verma [6] has investigated the harmonic thermoelastic waves and dispersion relations in layered anisotropic plate by utilizing the transfer matrix method.

Nowadays, composite-metal structures have developed significantly in the industry because of the great mechanical properties rather than other materials. Furthermore, damage detection using wave propagation is one of the most interesting topics that have attracted the attention of researchers. Thus, in this paper, we extend the relations of dispersion curves for multilayered composite-metal plates using the transfer matrix method presented in previous studies. Presented procedure can be used for damage detection in multilayered composite-metal structures using the wave propagation methods.

\section{Wave Propagation Formulation}

2.1. Wave Propagation in a Single-Layered Orthotropic Plate. Consider an orthotropic plate stacked normal to the axis 


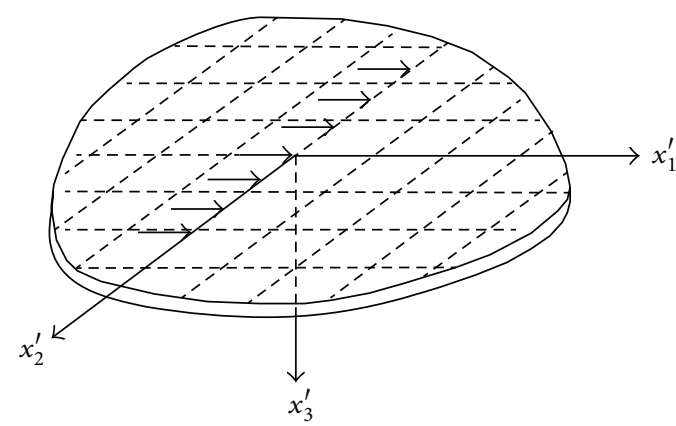

(a)

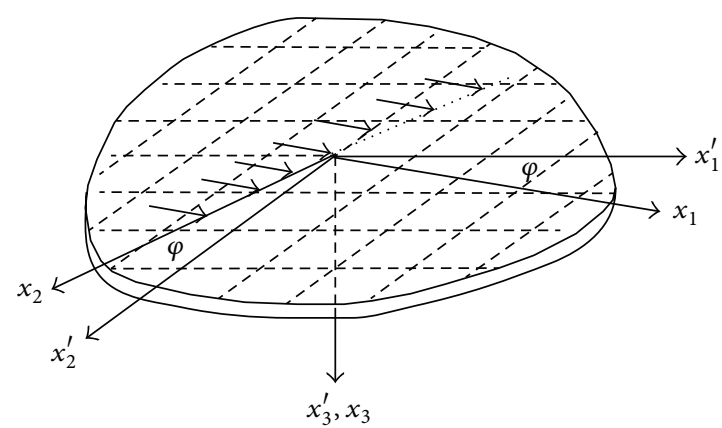

(b)

FIGURE 1: Wave propagation along (a) principle-axes and (b) off-principle-axes.

$x_{3}$ of the global coordinate system $\left(x_{1}, x_{2}, x_{3}\right)$ and consider $x_{1}-x_{2}$ plane coincides with the upper surface of the plate. Generally, the global coordinate system $\left(x_{1}, x_{2}, x_{3}\right)$ does not coincide with the principle material coordinate system $\left(x_{1}^{\prime}, x_{2}^{\prime}, x_{3}^{\prime}\right)$. In this paper two different cases are considered: wave propagation along principle-axes, $x_{1}^{\prime}$ or $x_{2}^{\prime}$, and wave propagation along off-principle-axes (see Figure 1).

2.1.1. Wave Propagation along Principle-Axes. With respect to the local coordinate system $\left(x_{1}^{\prime}, x_{2}^{\prime}, x_{3}^{\prime}\right)$, equations of motion are as follows:

$$
\begin{aligned}
& \frac{\partial \sigma_{11}^{\prime}}{\partial x_{1}^{\prime}}+\frac{\partial \sigma_{12}^{\prime}}{\partial x_{2}^{\prime}}+\frac{\partial \sigma_{13}^{\prime}}{\partial x_{3}^{\prime}}=\rho \frac{\partial^{2} u_{1}^{\prime}}{\partial t^{2}} \\
& \frac{\partial \sigma_{12}^{\prime}}{\partial x_{1}^{\prime}}+\frac{\partial \sigma_{22}^{\prime}}{\partial x_{2}^{\prime}}+\frac{\partial \sigma_{23}^{\prime}}{\partial x_{3}^{\prime}}=\rho \frac{\partial^{2} u_{2}^{\prime}}{\partial t^{2}} \\
& \frac{\partial \sigma_{13}^{\prime}}{\partial x_{1}^{\prime}}+\frac{\partial \sigma_{23}^{\prime}}{\partial x_{2}^{\prime}}+\frac{\partial \sigma_{33}^{\prime}}{\partial x_{3}^{\prime}}=\rho \frac{\partial^{2} u_{3}^{\prime}}{\partial t^{2}}
\end{aligned}
$$

and stress-strain relations are given by

$$
\left\{\begin{array}{l}
\sigma_{11}^{\prime} \\
\sigma_{22}^{\prime} \\
\sigma_{33}^{\prime} \\
\sigma_{23}^{\prime} \\
\sigma_{31}^{\prime} \\
\sigma_{12}^{\prime}
\end{array}\right\}=\left[\begin{array}{cccccc}
C_{11}^{\prime} & C_{12}^{\prime} & C_{13}^{\prime} & 0 & 0 & 0 \\
C_{12}^{\prime} & C_{22}^{\prime} & C_{23}^{\prime} & 0 & 0 & 0 \\
C_{13}^{\prime} & C_{23}^{\prime} & C_{33}^{\prime} & 0 & 0 & 0 \\
0 & 0 & 0 & C_{44}^{\prime} & 0 & 0 \\
0 & 0 & 0 & 0 & C_{55}^{\prime} & 0 \\
0 & 0 & 0 & 0 & 0 & C_{66}^{\prime}
\end{array}\right]\left\{\begin{array}{c}
\varepsilon_{11}^{\prime} \\
\varepsilon_{22}^{\prime} \\
\varepsilon_{33}^{\prime} \\
\varepsilon_{23}^{\prime} \\
\varepsilon_{31}^{\prime} \\
\varepsilon_{12}^{\prime}
\end{array}\right\},
$$

where $\sigma^{\prime}, \varepsilon^{\prime}, u^{\prime}$ are the components of stress, strain, and displacement, respectively, and $\rho$ and $C^{\prime}$ are the material density and stiffness coefficient, respectively.

Furthermore, the linear strain-displacement relations are

$$
\begin{gathered}
\varepsilon_{11}^{\prime}=\frac{\partial u_{1}^{\prime}}{\partial x_{1}^{\prime}}, \quad \varepsilon_{22}^{\prime}=\frac{\partial u_{2}^{\prime}}{\partial x_{2}^{\prime}}, \quad \varepsilon_{33}^{\prime}=\frac{\partial u_{3}^{\prime}}{\partial x_{3}^{\prime}} \\
\varepsilon_{23}^{\prime}=\frac{\partial u_{2}^{\prime}}{\partial x_{3}^{\prime}}+\frac{\partial u_{3}^{\prime}}{\partial x_{2}^{\prime}}, \quad \varepsilon_{31}^{\prime}=\frac{\partial u_{1}^{\prime}}{\partial x_{3}^{\prime}}+\frac{\partial u_{3}^{\prime}}{\partial x_{1}^{\prime}} \\
\varepsilon_{12}^{\prime}=\frac{\partial u_{2}^{\prime}}{\partial x_{1}^{\prime}}+\frac{\partial u_{1}^{\prime}}{\partial x_{2}^{\prime}} .
\end{gathered}
$$

Substituting (2) and (3) into (1a), (1b), and (1c) concludes the three following equations:

$$
\begin{gathered}
C_{11}^{\prime} \frac{\partial^{2} u_{1}^{\prime}}{\partial x_{1}^{\prime 2}}+C_{55}^{\prime} \frac{\partial^{2} u_{1}^{\prime}}{\partial x_{3}^{\prime 2}}+\left(C_{13}^{\prime}+C_{55}^{\prime}\right) \frac{\partial^{2} u_{3}^{\prime}}{\partial x_{1}^{\prime} \partial x_{3}^{\prime}}=\rho \frac{\partial^{2} u_{1}^{\prime}}{\partial t^{2}}, \\
C_{66}^{\prime} \frac{\partial^{2} u_{2}^{\prime}}{\partial x_{1}^{\prime 2}}+C_{44}^{\prime} \frac{\partial^{2} u_{2}^{\prime}}{\partial x_{3}^{\prime 2}}=\rho \frac{\partial^{2} u_{2}^{\prime}}{\partial t^{2}}, \\
C_{33}^{\prime} \frac{\partial^{2} u_{3}^{\prime}}{\partial x_{3}^{\prime 2}}+C_{55}^{\prime} \frac{\partial^{2} u_{3}^{\prime}}{\partial x_{1}^{\prime 2}}+\left(C_{13}^{\prime}+C_{55}^{\prime}\right) \frac{\partial^{2} u_{1}^{\prime}}{\partial x_{1}^{\prime} \partial x_{3}^{\prime}}=\rho \frac{\partial^{2} u_{3}^{\prime}}{\partial t^{2}} .
\end{gathered}
$$

Two coupled equations, (4a) and (4c), are related to pressure wave ( $\mathrm{P}$ wave) and shear vertical wave (SV wave). Also, the uncoupled equation (4b) is related to horizontal wave ( $\mathrm{SH}$ wave). Formal solutions for these equations are assumed as follows [3]:

$$
u_{j}^{\prime}=U_{j} e^{i k\left(x_{1}^{\prime}+\beta x_{3}^{\prime}-c t\right)}, \quad j=1,2,3,
$$

where $U_{j}$ and $c$ are displacement amplitude along direction $x_{j}^{\prime}$ and phase velocity in direction $x_{1}^{\prime}$, respectively, and $k(=\omega / c), \omega$, and $\beta$ are $x_{1}^{\prime}$-component of wavenumber and ratio of $x_{3}^{\prime}$, circular frequency, and $x_{1}^{\prime}$-components of wavenumber, respectively. For clarification, the rest of the solution procedure for coupled and uncoupled equations is described next.

(a) Solution for $\mathrm{SH}$ wave: according to (5), it is assumed the solution of (4b) has the following form:

$$
u_{2}^{\prime}=U_{2} e^{i k\left(x_{1}^{\prime}+\beta x_{3}^{\prime}-c t\right)}
$$

substituting (6) into (4b) results in

$$
\beta_{1}=-\beta_{2}=\left[\frac{\left(\rho c^{2}-C_{66}^{\prime}\right)}{C_{44}^{\prime}}\right]^{1 / 2}
$$


therefore

$$
u_{2}^{\prime}=U_{21} e^{i k\left(x_{1}^{\prime}+\beta_{1} x_{3}^{\prime}-c t\right)}+U_{22} e^{i k\left(x_{1}^{\prime}+\beta_{2} x_{3}^{\prime}-c t\right)} .
$$

Also, stress-strain relation is

$$
\sigma_{23}^{\prime}=C_{44}^{\prime} \frac{\partial u_{2}^{\prime}}{\partial x_{3}^{\prime}}
$$

thus

$$
\sigma_{23}^{\prime}=i k C_{44}^{\prime}\left[\beta_{1} U_{21} e^{i k\left(x_{1}^{\prime}+\beta_{1} x_{3}^{\prime}-c t\right)}+\beta_{2} U_{22} e^{i k\left(x_{1}^{\prime}+\beta_{2} x_{3}^{\prime}-c t\right)}\right] .
$$

Using (8) and (10) and after some simplifications, stress and displacement at $x_{3}^{\prime}=0$ may be related to those at $x_{3}^{\prime}=h$ ( $h$ is thickness of plate) by following the relation

$$
\left[\begin{array}{c}
u_{2}^{\prime} \\
\sigma_{23}^{*}
\end{array}\right]_{x_{3}^{\prime}=h}=[T]\left[\begin{array}{c}
u_{2}^{\prime} \\
\sigma_{23}^{*}
\end{array}\right]_{x_{3}^{\prime}=0}, \quad \sigma_{23}^{*}=\frac{\sigma_{23}^{\prime}}{i k},
$$

where $[T]$ is transfer matrix and is given by

$$
[T]=\left[\begin{array}{cc}
\cos (\beta k h) & \frac{i}{\left(\beta C_{44}^{\prime}\right)} \sin (\beta k h) \\
i \beta C_{44}^{\prime} \sin (\beta k h) & \cos (\beta k h)
\end{array}\right] .
$$

By invoking free stress boundary conditions at lower and upper surfaces of plate, characteristic equation for singlelayered plate may be obtained as follows:

$$
T_{21}=0 \Longrightarrow \sin (\beta k h)=0 .
$$

Substituting $k=\omega / c$ into (13) results in an equation with two unknown parameters $\omega$ and $c$ called "dispersion equation of SH wave."

(b) Solution for $\mathrm{P}$ and SV waves: according to (5), suggested solutions of (4a) and (4c) are

$$
u_{1}^{\prime}=U_{1} e^{i k\left(x_{1}^{\prime}+\beta x_{3}^{\prime}-c t\right)}, \quad u_{3}^{\prime}=U_{3} e^{i k\left(x_{1}^{\prime}+\beta x_{3}^{\prime}-c t\right)} .
$$

Substituting (14) into (4a) and (4c) results in

$$
\left[\begin{array}{cc}
C_{11}-\rho c^{2}+C_{55} \beta^{2} & \left(C_{13}+C_{55}\right) \beta \\
\left(C_{13}+C_{55}\right) \beta & C_{55}-\rho c^{2}+C_{33} \beta^{2}
\end{array}\right]\left\{\begin{array}{l}
U_{1} \\
U_{3}
\end{array}\right\}=0
$$

To obtain nontrivial solution, determinant of coefficients matrix of displacement vector is vanished. Therefore, a fourdegree polynomial equation in $\beta$ is obtained as follows:

$$
A \beta^{4}+B \beta^{2}+C=0
$$

There are four solutions for $\beta$ or in other words two pairs of solutions for $\beta^{2}$ as follows:

$$
\beta_{1}=-\beta_{2}, \quad \beta_{3}=-\beta_{4} ;
$$

therefore

$$
u_{1}^{\prime}=\sum_{j=1}^{4} U_{1 j} e^{i k\left(x_{1}^{\prime}+\beta_{j} x_{3}^{\prime}-c t\right)}, \quad u_{3}^{\prime}=\sum_{j=1}^{4} U_{3 j} e^{i k\left(x_{1}^{\prime}+\beta_{j} x_{3}^{\prime}-c t\right)} .
$$

Also, stress-strain relation is

$$
\sigma_{33}^{\prime}=C_{13}^{\prime} \frac{\partial u_{1}^{\prime}}{\partial x_{1}^{\prime}}+C_{33}^{\prime} \frac{\partial u_{3}^{\prime}}{\partial x_{3}^{\prime}}, \quad \sigma_{13}^{\prime}=C_{55}^{\prime}\left(\frac{\partial u_{3}^{\prime}}{\partial x_{1}^{\prime}}+\frac{\partial u_{1}^{\prime}}{\partial x_{3}^{\prime}}\right) ;
$$

thus

$$
\begin{aligned}
& \sigma_{33}^{\prime}=\sum_{j=1}^{4} i k D_{1 j} U_{1 j} e^{i k\left(x_{1}^{\prime}+\beta_{j} x_{3}^{\prime}-c t\right)}, \\
& \sigma_{13}^{\prime}=\sum_{j=1}^{4} i k D_{2 j} U_{1 j} e^{i k\left(x_{1}^{\prime}+\beta_{j} x_{3}^{\prime}-c t\right)},
\end{aligned}
$$

where

$$
\begin{gathered}
D_{1 j}=C_{13}^{\prime}+C_{33}^{\prime} \beta_{j} W_{j}, \\
D_{2 j}=C_{55}^{\prime}\left(\beta_{j}+W_{j}\right), \\
W_{j}=\frac{U_{3 j}}{U_{1 j}}=\frac{\rho c^{2}-C_{11}^{\prime}-C_{55}^{\prime} \beta_{j}^{2}}{\left(C_{13}^{\prime}+C_{55}^{\prime}\right) \beta_{j}} .
\end{gathered}
$$

Using (18), (20), and (21) and after some simplifications, stress and displacement at $x_{3}^{\prime}=0$ may be related to those at $x_{3}^{\prime}=h$ ( $h$ is thickness of plate) by following the relation

$$
\left[\begin{array}{llll}
u_{1}^{\prime} & u_{3}^{\prime} & \sigma_{33}^{\prime} & \sigma_{13}^{\prime}
\end{array}\right]_{x_{3}^{\prime}=h}^{t}=[T]\left[\begin{array}{llll}
u_{1}^{\prime} & u_{3}^{\prime} & \sigma_{33}^{\prime} & \sigma_{13}^{\prime}
\end{array}\right]_{x_{3}^{\prime}=0}^{t}
$$

where $[T]$ is transfer matrix and is given by

$$
\begin{gathered}
{[T]=T_{1} T_{2} T_{1}^{-1},} \\
T_{1}=\left[\begin{array}{cccc}
1 & 1 & 1 & 1 \\
W_{1} & -W_{1} & W_{3} & -W_{3} \\
D_{11} & D_{11} & D_{13} & D_{13} \\
D_{21} & -D_{21} & D_{23} & -D_{23}
\end{array}\right], \\
T_{2}=\operatorname{diag}\left(e^{i k \beta_{j} h}\right), \quad j=1,2,3,4 .
\end{gathered}
$$

By invoking free stress boundary conditions at lower and upper surfaces of plate, characteristic equation for singlelayered plate may be obtained as follows:

$$
\left|\begin{array}{ll}
T_{31} & T_{32} \\
T_{41} & T_{42}
\end{array}\right|=0
$$

Substituting $k=\omega / c$ into (24) results in an equation with two unknown parameters $\omega$ and $c$ called "dispersion equation of $\mathrm{P}$ and SV waves."

2.1.2. Wave Propagation along Off-Principle-Axes. Assume wave propagation along off-principle-axis $x_{1}$ (or $x_{2}$ ) with angle $\varphi$ with respect to principle-axis $x_{1}^{\prime}$ (or $x_{2}^{\prime}$ ) as shown in Figure 1(b). In this situation, (4a), (4b), and (4c) are coupled for all materials except isotropic one. The reason is that 
transformed stiffness matrix from system $\left(x_{1}^{\prime}, x_{2}^{\prime}, x_{3}^{\prime}\right)$ into system $\left(x_{1}, x_{2}, x_{3}\right)$ has a monoclinic form as follows:

$$
C=\left[\begin{array}{cccccc}
C_{11} & C_{12} & C_{13} & 0 & 0 & C_{16} \\
C_{12} & C_{22} & C_{23} & 0 & 0 & C_{26} \\
C_{13} & C_{23} & C_{33} & 0 & 0 & C_{36} \\
0 & 0 & 0 & C_{44} & C_{45} & 0 \\
0 & 0 & 0 & C_{45} & C_{55} & 0 \\
C_{16} & C_{26} & C_{36} & 0 & 0 & C_{66}
\end{array}\right]
$$

Substituting (5) into (1a), (1b), and (1c) results in

$$
\begin{aligned}
{[K(\beta)] U_{i} } & =0, \\
K_{11} & =C_{11}-\rho c^{2}+C_{55} \beta^{2}, \\
K_{12} & =C_{16}+C_{45} \beta^{2}, \\
K_{13} & =\left(C_{13}+C_{55}\right) \beta, \\
K_{22} & =C_{66}-\rho c^{2}+C_{44} \beta^{2}, \\
K_{23} & =\left(C_{36}+C_{45}\right) \beta, \\
K_{33} & =C_{55}-\rho c^{2}+C_{33} \beta^{2} .
\end{aligned}
$$

By setting determinant $[K(\beta)]$ equal to zero, a six-degree polynomial equation is obtained as follows:

$$
A \beta^{6}+B \beta^{4}+C \beta^{2}+D=0 .
$$

There are four solutions for $\beta$ as follows:

$$
\beta_{1}=-\beta_{2}, \quad \beta_{3}=-\beta_{4}, \quad \beta_{5}=-\beta_{6} .
$$

Therefore, displacements and stress components are given by

$$
\begin{gathered}
\left(u_{1}, u_{2}, u_{3}\right)=\sum_{j=1}^{6}\left(1, V_{j}, W_{j}\right) U_{1 j} e^{i k\left(x_{1}+\beta_{j} x_{3}-c t\right)}, \\
\left(\sigma_{33}, \sigma_{13}, \sigma_{23}\right)=\sum_{j=1}^{4}\left(D_{1 j}, D_{2 j}, D_{3 j}\right) i k U_{1 j} e^{i k\left(x_{1}+\beta_{j} x_{3}-c t\right)},
\end{gathered}
$$

where

$$
\begin{gathered}
D_{1 j}=C_{13}+C_{36} V_{j}+C_{33} \beta_{j} W_{j}, \\
D_{2 j}=C_{55}\left(\beta_{j}+W_{j}\right)+C_{45} \beta_{j} V_{j}, \\
D_{3 j}=C_{45}\left(\beta_{j}+W_{j}\right)+C_{44} \beta_{j} V_{j}, \\
V_{j}=\frac{U_{2 j}}{U_{1 j}}=\frac{K_{11} K_{23}-K_{13} K_{12}}{K_{13} K_{22}-K_{12} K_{23}}, \\
W_{j}=\frac{U_{3 j}}{U_{1 j}}=\frac{K_{11} K_{23}-K_{13} K_{12}}{K_{33} K_{22}-K_{13} K_{23}} .
\end{gathered}
$$

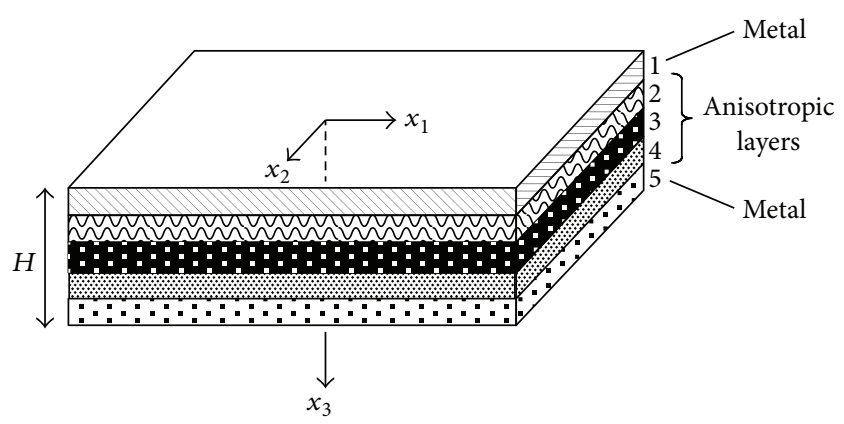

FIGURE 2: Multilayered plate.

Using (29) and (30) and after some simplifications, stress and displacement at $x_{3}=0$ may be related to those at $x_{3}=x_{3}^{\prime}=h$ ( $h$ is thickness of plate) by following the relation

$$
\begin{gathered}
{\left[\begin{array}{llllll}
u_{1} & u_{2} & u_{3} & \sigma_{33}^{*} & \sigma_{13}^{*} & \sigma_{23}^{*}
\end{array}\right]_{x_{3}^{\prime}=h}^{t}} \\
=[T]\left[\begin{array}{llllll}
u_{1} & u_{2} & u_{3} & \sigma_{33}^{*} & \sigma_{13}^{*} & \sigma_{23}^{*}
\end{array}\right]_{x_{3}^{\prime}=0}^{t}, \\
\sigma_{m n}^{*}=\frac{\sigma_{m n}}{i k}, \quad m, n=1,2,3,
\end{gathered}
$$

where $[T]$ is transfer matrix and is given by

$$
\begin{gathered}
{[T]=T_{1} T_{2} T_{1}^{-1},} \\
T_{1}=\left[\begin{array}{cccccc}
1 & 1 & 1 & 1 & 1 & 1 \\
V_{1} & V_{1} & V_{3} & V_{3} & V_{5} & V_{5} \\
W_{1} & -W_{1} & W_{3} & -W_{3} & W_{5} & -W_{5} \\
D_{11} & D_{11} & D_{13} & D_{13} & D_{15} & D_{15} \\
D_{21} & -D_{21} & D_{23} & -D_{23} & D_{25} & -D_{25} \\
D_{31} & -D_{31} & D_{33} & -D_{33} & D_{35} & -D_{35}
\end{array}\right], \\
T_{2}=\operatorname{diag}\left(e^{i k \beta_{j} h}\right), \quad j=1,2, \ldots, 6 .
\end{gathered}
$$

By invoking free stress boundary conditions at lower and upper surfaces of plate, characteristic equation for singlelayered plate may be obtained as follows:

$$
\left|\begin{array}{lll}
T_{41} & T_{42} & T_{43} \\
T_{51} & T_{52} & T_{53} \\
T_{61} & T_{62} & T_{63}
\end{array}\right|=0
$$

Substituting $k=\omega / c$ into (33) results in an equation with two unknown parameters $\omega$ and $c$.

Note 1. All of presented relations may be used for transversely isotropic, cubic, and isotropic materials, whereas (1a), (1b), and (1c)-(24) are enough for isotropic material because any arbitrary propagation direction coincides with one of the material symmetry axes!

Note 2. The presented procedure in Section 2.1.2 may be used for wave analysis in any direction of monoclinic plates.

2.2. Wave Propagation in a Multilayered Composite-Metal Plate. The above procedure for single-layered plate may 


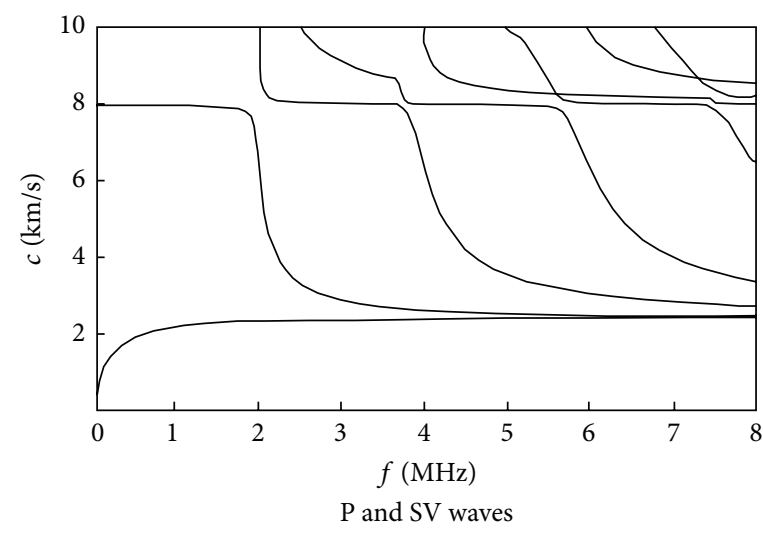

(a)

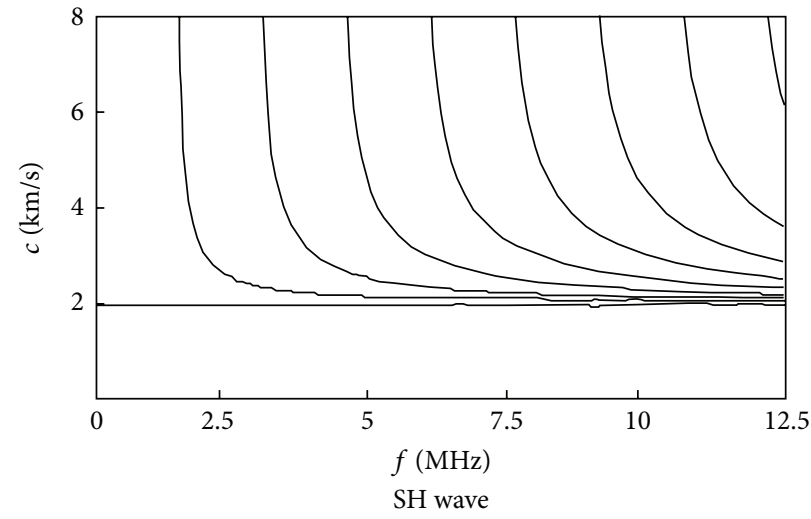

(b)

FIGURE 3: Wave propagation along principle-axes of a single-layered orthotropic plate.

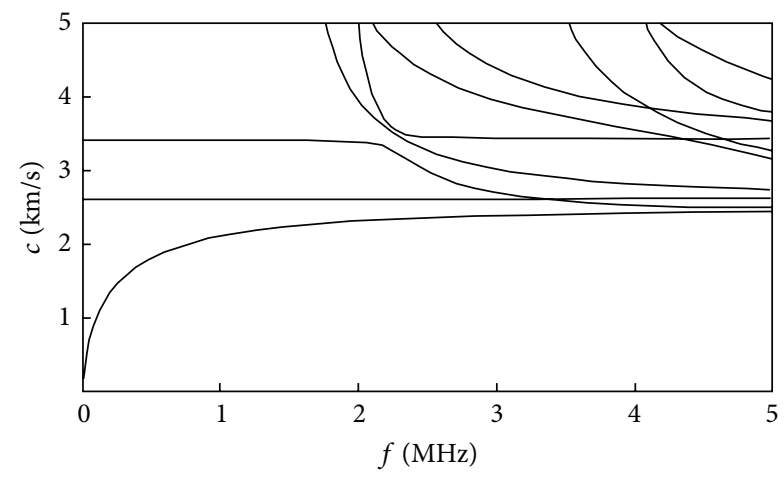

FigURE 4: Wave propagation along off-principle-axes $\left(\varphi=30^{\circ}\right)$ of a single-layered orthotropic plate ( $\mathrm{SH}, \mathrm{SV}$, and $\mathrm{P}$ waves).

be extended for a multilayered composite-metal plate (see Figure 2) by "transform matrix method." In this method by applying the continuity of the displacement and stress components at the layer interfaces of plate, displacements and stress components at lower surface can be related to those at upper one. For instance, in Figure 2, transfer matrix between $x_{3}=0$ and $x_{3}=H$ is given by

$$
T_{\text {Multi }}=T_{1 m} T_{2 a} T_{3 a} T_{4 a} T_{5 m},
$$

where $m$ and $a$ refer to metal layer and anisotropic composite layer, respectively. By satisfying appropriate boundary condition on the outer boundaries, wave characteristic equations are obtained. It is noteworthy that a local coordinate system $\left(x_{1}^{\prime}, x_{2}^{\prime}, x_{3}^{\prime}\right)_{p}$ is considered for each layer $p$ to obtain $T_{p}$ using the presented equations in Section 2.1.

2.3. Dispersion Curves of Waves. Presented dispersion equations of waves in previous section such as (13), (24), and (33) may be used for plotting of dispersion curves of phase velocity $c$ as function of frequency $f(=\omega / 2 \pi)$. These curves are important tools in damage detection techniques based on the wave propagation. However, the dispersion equations of waves do not generally have analytical solution and can be solved by numerical methods.

\section{Numerical Examples}

In this section, some numerical examples for calculation of dispersion curves in composite materials are presented in order to verify and validate the mentioned procedure. Two different cases are considered: wave propagation along principle- and off-principle-axes in (a) single-layered orthotropic plate and (b) two-layered composite-metal plate. We use MATLAB program to solve dispersion equation numerically. In the sequel, we elucidate each case study.

3.1. Dispersion Curves of Waves in Single-Layered Orthotropic Plate. In this subsection, dispersion curves of waves are plotted for a single-layered orthotropic plate with the following material properties: $\rho=2 \mathrm{gr} / \mathrm{cm}^{3}, h=1 \mathrm{~mm}, C_{11}^{\prime}=$ $128, C_{12}^{\prime}=7, C_{13}^{\prime}=6, C_{22}^{\prime}=72, C_{23}^{\prime}=5, C_{33}^{\prime}=32, C_{44}^{\prime}=$ $18, C_{55}^{\prime}=12.25, C_{66}^{\prime}=8, C_{11}^{\prime}=2(\mathrm{GPa})$.

Two different cases are considered: (a) wave propagates along principle-axis $x_{1}^{\prime}$ in which (13) and (24) are used for SH wave and P and SV waves, respectively (see Figure 3); (b) wave propagates along off-principle-axis $x_{1}$ with angle $\varphi=$ $30^{\circ}$ with respect to $x_{1}^{\prime}$ in which (33) are used for $\mathrm{SH}, \mathrm{P}$, and $\mathrm{SV}$ waves as shown in Figure 4.

3.2. Dispersion Curves of Waves in Composite-Metal Plate. To verify the mentioned procedure in Section 2.2, we illustrate the wave dispersion curves in a double-layered plate including an orthotropic layer with the same material properties listed in Section 3.1 and an aluminum layer with the following material properties:

$$
\begin{array}{cl}
E=70 \mathrm{GPa}, & \nu=0.3, \\
\rho=2.7 \mathrm{gr} / \mathrm{cm}^{3}, & h=1 \mathrm{~mm} .
\end{array}
$$

In this situation, according to (34), transfer matrix is = $T_{\mathrm{Al}} T_{\text {orth }}$, where $T_{\text {orth }}$ and $T_{\mathrm{Al}}$ are calculated using (24) if 

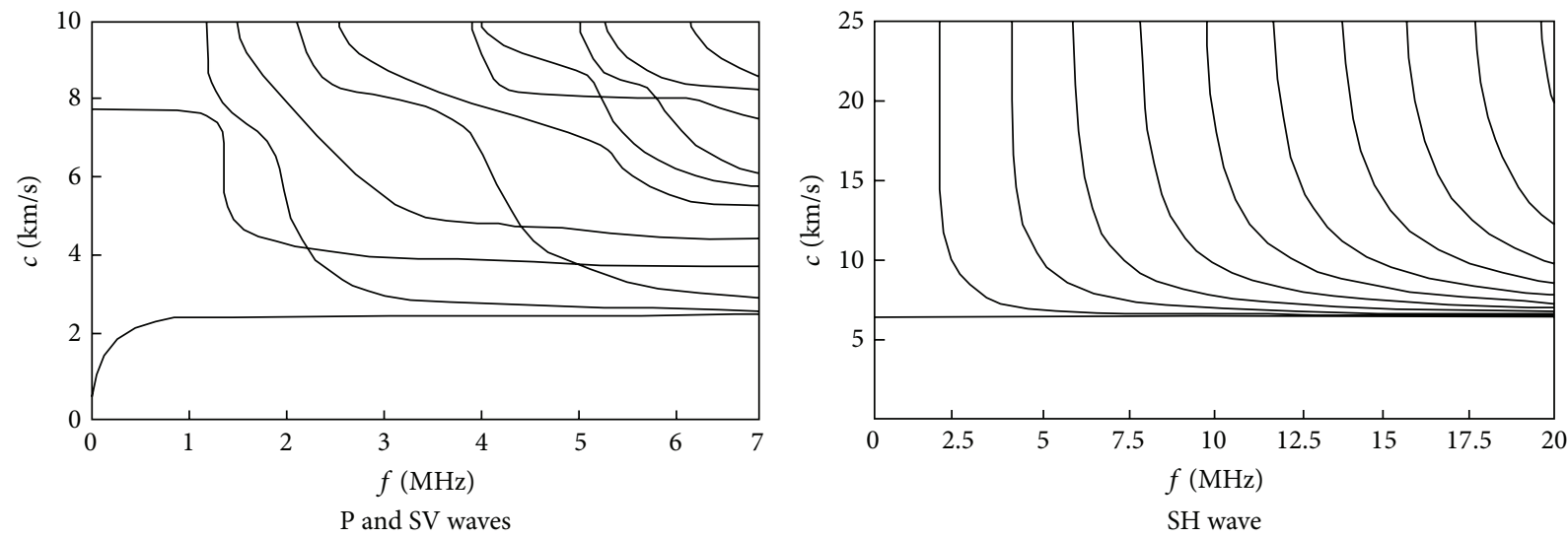

FIGURE 5: Wave propagation along principle-axes of a double-layered Al-orthotropic plate.

wave propagates along principle-axes of orthotropic layer. Dispersion curves of SH wave and P and SV waves are shown in Figure 5.

\section{Conclusion}

In this paper, wave dispersion relations of single anisotropic layer were presented. Two different cases were considered: propagation of wave along an axis of material symmetry and along off-axes of material symmetry. Since compositemetal structures have developed in last years, presented equations were extended for a multilayered composite-metal plate using the transfer matrix method. Suggested procedure is straightforward and applicable for damage detection based on wave propagation. However, the cost of calculations increases for large number of layers.

\section{Conflict of Interests}

The authors declare that there is no conflict of interests regarding the publication of this paper.

\section{References}

[1] V. Giurgiutiu, Structural Health Monitoring with Piezoelectric Wafer Active Sensors, Elsevier Academic Press, New York, NY, USA, 2008.

[2] V. Giurgiutiu, J. Bao, and W. Zhao, "Piezoelectric wafer active sensor embedded ultrasonics in beams and plates," Experimental Mechanics, vol. 43, no. 4, pp. 428-449, 2003.

[3] A. H. Nayfeh, "The general problem of elastic wave propagation in multilayered anisotropic media," The Journal of the Acoustical Society of America, vol. 89, no. 4, pp. 1521-1531, 1991.

[4] A. Demcenko and L. Mazeika, "Calculation of lamb waves dispersion curves in multi-layered planar structures," Journal of Sound and Vibration, vol. 329, no. 9, pp. 1435-1449, 2010.

[5] M. J. S. Lowe, "Matrix techniques for modeling ultrasonic waves in multilayered media," IEEE Transactions on Ultrasonics, Ferroelectrics, and Frequency Control, vol. 42, no. 4, pp. 525-542, 1995.
[6] K. L. Verma, "The general problem of thermoelastic wave propagation in multi-layered anisotropic media with application to periodic media," International Journal of Applied Engineering, vol. 1, no. 4, pp. 908-922, 2011. 

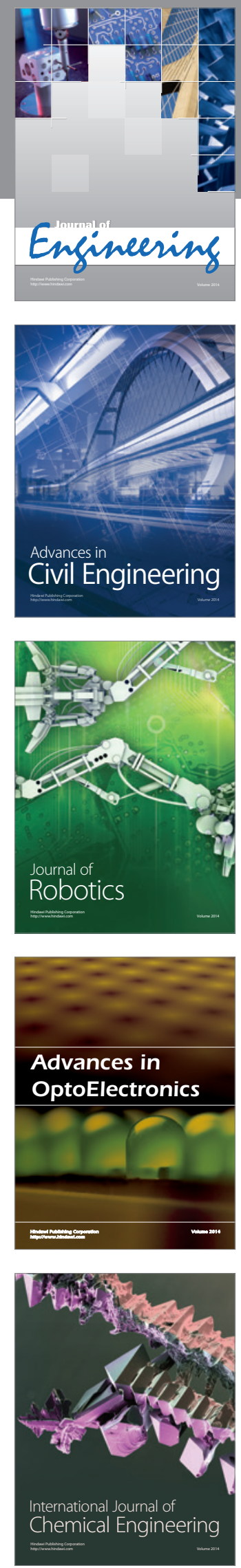

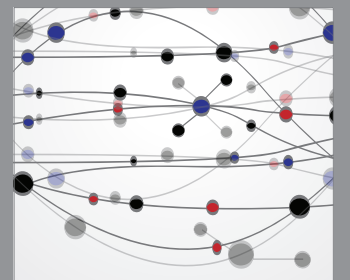

The Scientific World Journal
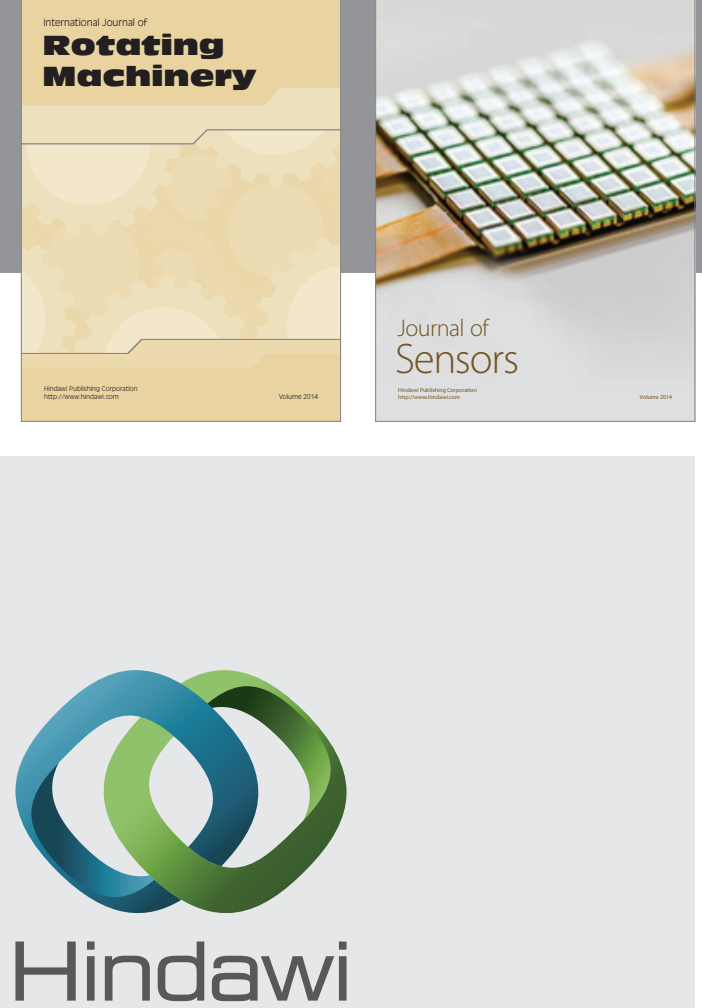

Submit your manuscripts at http://www.hindawi.com
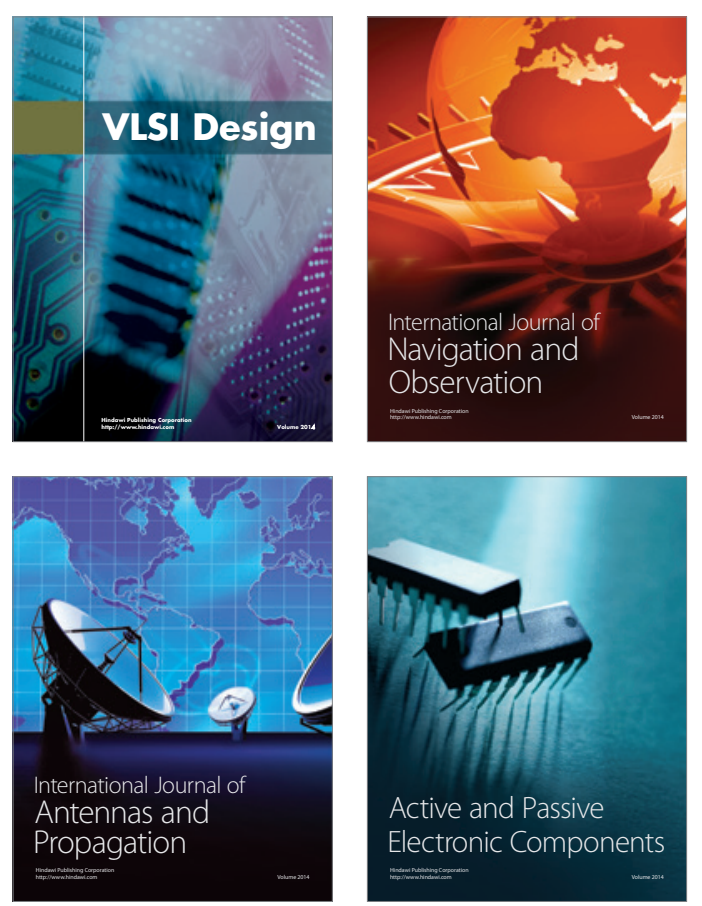
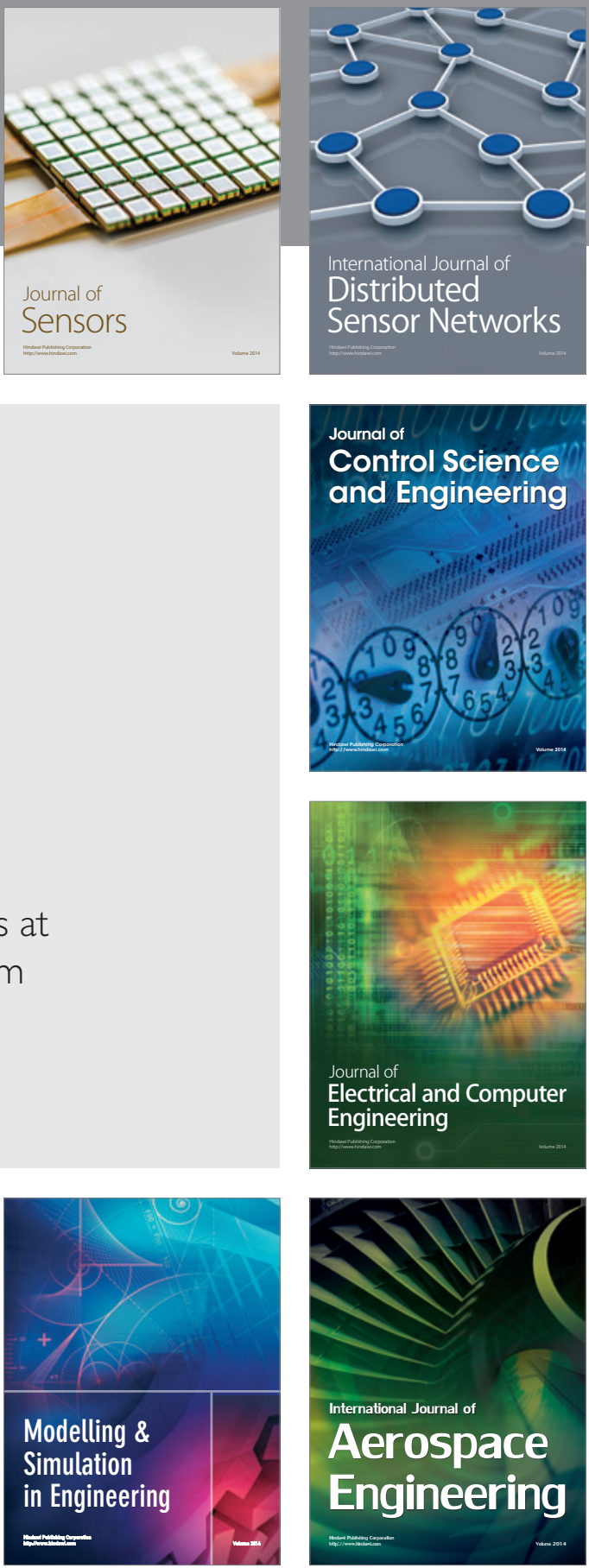

Journal of

Control Science

and Engineering
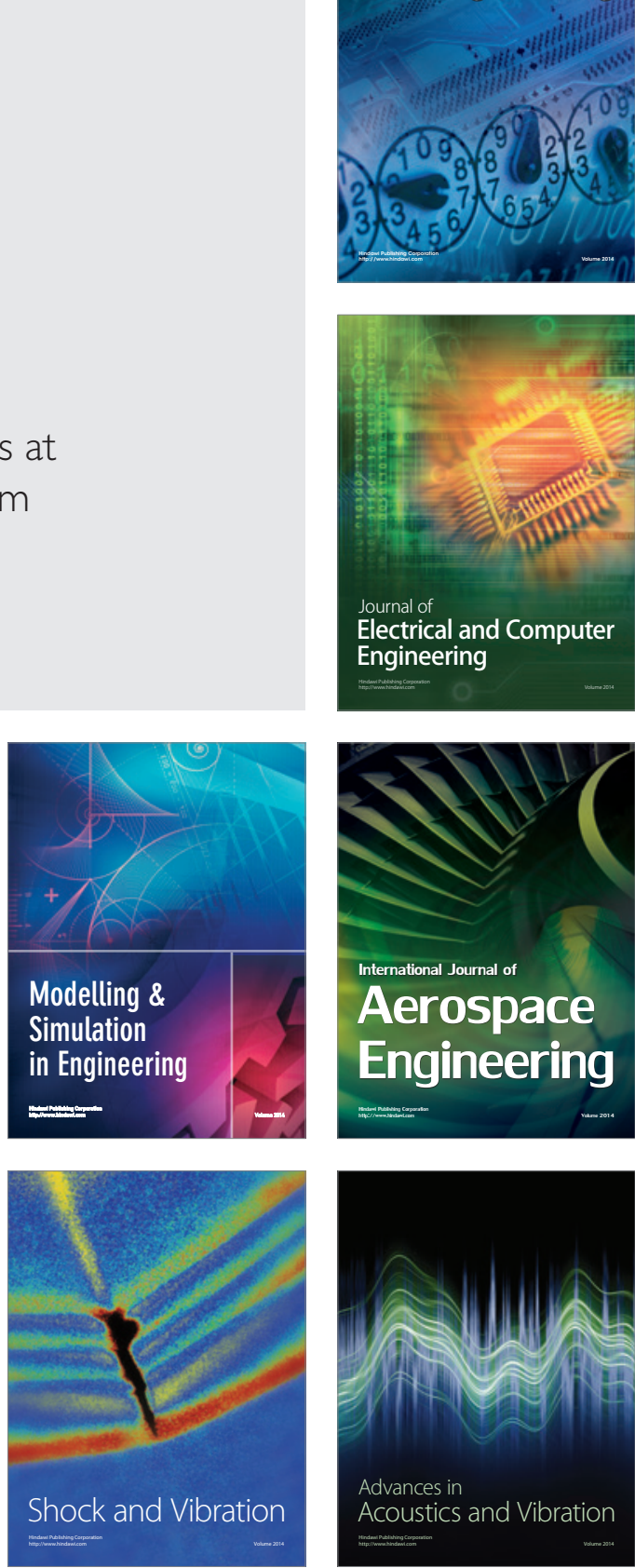